\title{
IUFOST2006/802 Optimising a multi-deck refrigerated display cabinet using CFD
}

\author{
A. Foster, M. Madge and J. Evans \\ University of Bristol (FRPERC), Churchill Building, Langford, BS40 5DU Bristol, United Kingdom \\ j.a.evans@bristol.ac.uk
}

In the UK total sales of chilled food grew by $18.4 \%$ between 1999 and 2003, to a value of $£ 7.68 \mathrm{bn}$. Refrigeration is the largest load in a supermarket and increasingly stringent demands are being placed on manufacturers to develop refrigerated retail display cabinets capable of achieving close temperature control with low energy consumption. Subtle changes to the airflow of open fronted multi-deck cabinets can have dramatic effects on both the product temperature and energy consumption. Studies were carried out to see if computational fluid dynamic (CFD) modelling could be used to rapidly identify the changes required to an existing cabinet so that it would meet a higher test specification. Method All tests were carried out in a test room conforming to EN441 standards and climate class III (temperature of $25^{\circ} \mathrm{C}$ and relative humidity of $60 \%$ ). It was not feasible to create a CFD model of the whole cabinet that included the necessary detail with which to troubleshoot the cabinet performance failings. Instead CFD modelling concentrated specifically on two areas of the cabinet that were most likely to produce the regions of high temperature. 1 . The flow of air as it left the evaporator and traveled up the rear duct at the corners of the cabinet. 2. The flow of air as it left the air curtain and the rear grille and its interaction with product on the top shelf. Results Before optimisation, only 42 out of the 54 measurement packs spent the entire test period between the specified temperatures limits $\left(-1\right.$ and $\left.4^{\circ} \mathrm{C}\right)$. After optimisation this increased to 53 measurement packs. Also the average power consumption reduced from 1.37 to $1.29 \mathrm{~kW}$ after modification. Conclusions The use of CFD to understand the problem and test possible solutions was invaluable in improving the cabinet. Many authors have proved CFD's benefits to predict air flow in display cabinets; however, this work has generally been carried out during research projects lasting a number of months or even years. The benefit to the display cabinet manufacture is to get results quickly (within a couple of weeks), this work has proved this possible. 